\title{
IMAGEM CORPORAL E SUA RELAÇÃO COM PESO E ÍNDICE DE MASSA CORPORAL EM BAILARINOS PROFISSIONAIS
}

\author{
BODY IMAGE AND ITS RELATIONSHIP TO WEIGHT AND BODY MASS INDEX IN \\ PROFESSIONAL DANCERS
}

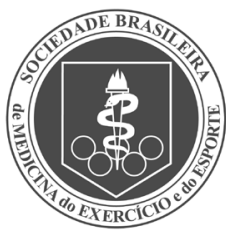

Artigo Original

\author{
IMAGEN CORPORAL Y SU RELACIÓN COM EL PESO E ÍNDICE DE MASA \\ CORPORAL EN BAILARINES PROFESIONALES
}

\begin{abstract}
Joseani Paulini Neves Simas ${ }^{1}$ (Educadora Física)

Ana Macara ${ }^{2}$

(Educadora Física)

Sebastião Iberes Lopes Melo

(Educador Físico)

1. Departamento de Educação Física do Centro de Ciências da Saúde e do Esporte (CEFID) da Universidade do Estado de Santa Catarina (UDESC), Florianópolis, SC, Brasil.

2. Centro de Estudos em Artes Performativas da Faculdade de Motricidade Humana (FMH) da Universidade de Lisboa (UL),

Lisboa, Portugal.
\end{abstract}

\section{Correspondência:}

Av. Trompowsky, 227, apto 1201,

Florianópolis, SC, Brasil. 88015-300.

joseanisimas@gmail.com

\begin{abstract}
RESUMO
Introdução: A imagem corporal é fenômeno multidimensional, dinâmico e vinculado ao corpo em movimento. Uma vez que o corpo é o instrumento de expressão na dança, dependendo da técnica de dança adotada pelo bailarino, demanda por um padrão estético, podendo estar relacionado à satisfação com a imagem corporal. Objetivo: Investigar a satisfação com a imagem corporal e sua relação com a percepção do peso e o índice de massa corporal em bailarinos profissionais brasileiros. Métodos: Foram estudados 181 bailarinos (105 clássicos e 76 contemporâneos) de companhias de dança da elite brasileira. As variáveis sociodemográficas, da prática e antropométricas foram obtidas por meio de informações autoreferidas. Utilizou-se a Escala de Silhuetas de Stunkard para avaliação da satisfação da imagem corporal. Os dados foram analisados utilizando-se a estatística descritiva (frequência, média e desvio padrão) e inferencial não paramétrica (testes do Qui-quadrado, Spearman e U de Mann-Whitney). Em todas as análises adotou-se $p \leq 0,05$. Resultados: Os bailarinos de ambas as técnicas de dança estão com IMC dentro da faixa da normalidade; houve diferenças estatísticas do IMC, percepção do peso e satisfação com a imagem corporal entre as técnicas de dança investigadas, estando os clássicos insatisfeitos por excesso de peso, desejando pesar menos, enquanto que os contemporâneos parecem estar satisfeitos e desejam pesar mais; os valores das correlações entre a satisfação da imagem corporal com IMC e com PPC foram baixos, somente havendo correlação negativa da SIC com IMC na dança contemporânea. Conclusão: Destaca-se que a técnica de dança adotada pelos bailarinos profissionais e padrões antropométricos pode estar relacionada a satisfação com a imagem corporal.
\end{abstract}

Palavras-chave: imagem corporal, dança, satisfação pessoal.

\section{ABSTRACT}

Introduction: Body image is multidimensional, dynamic phenomenon linked to the moving body. Once the body is the instrument of expression in dance, depending on the technique adopted by the dancer it requires an aesthetic standard that can be related to satisfaction with body image. Objective: To investigate the satisfaction with body image and its relationship with the perception of weight and body mass index in Brazilian professional dancers. Methods: One hundred and eighty one dancers (105 of classical and 76 of contemporary dance) of dance companies of the Brazilian elite were analyzed. The socio demographic, anthropometric and practice were obtained through self-reported information. We used the Stunkard's figure rating scale for assessment of body image satisfaction. Data were analyzed using descriptive statistics (frequency, mean and standard deviation) and inferential nonparametric (chi-square test, Spearman and Mann-Whitney). In all analyzes $p \leq 0.05$ was adopted. Results: The dancers of both techniques have a BMI within the normal range; statistical differences in BMI, weight perception and satisfaction with body image between dance techniques investigated, and classical dancers were dissatisfied with being overweight, wanting to have less weigh, while contemporary dancers seemed to be satisfied and want to weigh more; the values of the correlations between satisfaction with body image and BMI with PPC were lower, with only negative correlation between BMI and SIC in this group. Conclusion: It is noteworthy that the dance technique adopted by professional dancers and anthropometric patterns can be related to satisfaction with body image.

Keywords: body image, dancing, personal satisfaction.

\section{RESUMEN}

Introducción: La imagen corporal es un fenómeno multidimensional, dinámico y vinculado al cuerpo en movimiento. Dado que el cuerpo es el instrumento de expresión en la danza, dependiendo de la técnica de danza adoptada por el bailarín, demanda por un estándar estético, pudiendo estar relacionado a la satisfacción con la imagen corporal. Objetivo: Investigar la satisfacción con la imagen corporal y su relación con la percepción del peso y el índice de masa corporal en bailarines profesionales brasileños. Métodos: Fueron estudiados 181 bailarines (105 clásicos y 76 contemporáneos) de compañías de danza de la élite brasileña. Las variables sociodemográficas, de la práctica y antropométricas fueron obtenidas a través de informaciones autorreferidas. Se utilizó la Escala de Siluetas de Stunkard para evaluación de la satisfacción de la imagen corporal. Los datos fueron analizados utilizándose la estadística descriptiva (frecuencia, 
promedio y desvío estándar) e inferencial no paramétrica (tests del Chi-cuadrado, Spearman y U de Mann-Whitney). En todos los análisis se adoptó $p \leq 0,05$. Resultados: Los bailarines de ambas técnicas de danza están con IMC dentro de la gama de la normalidad; hubo diferencias estadísticas del IMC, percepción del peso y satisfacción con la imagen corporal entre las técnicas de danza investigadas, estando los clásicos insatisfechos por exceso de peso, deseando pesar menos, mientras que los contemporáneos parecen estar satisfechos y desean pesar más; los valores de las correlaciones entre la satisfacción de la imagen corporal con IMC y con PPC fueron bajos, solamente habiendo correlación negativa de la SIC con IMC en la danza contemporánea. Conclusión: Se destaca que la técnica de danza adoptada por los bailarines profesionales y estándares antropométricos puede estar relacionada a la satisfacción con la imagen corporal.

Palabras clave: imagen corporal, danza, satisfacción personal.

\section{INTRODUÇÃO}

A imagem corporal é fenômeno multidimensional, altamente dinâmico e totalmente vinculado ao corpo em movimento', definida no modo pelo qual o corpo se apresenta para cada indivíduo². Pode ser influenciada pelo sexo, idade, meios de comunicação, bem como pela relação do corpo com os processos cognitivos como crenças, atitudes e valores inseridos em uma cultura ${ }^{3}$.

O bailarino é um profissional de dedicação integral com demandas físicas comparáveis às de um atleta de alto rendimento ${ }^{4}$, onde sua identidade estética depende e compromete o seu desempenho técnico e artístico. Em virtude do foco constante no corpo, preocupações corporais são extremamente comuns entre bailarinos ${ }^{5}$. 0 trabalho corporal está sempre em evidência e faz parte de sua rotina ${ }^{6}$ dependendo do tempo de prática e idade dos bailarinos ${ }^{7}$, da intensidade das classes $^{8}$ e das pressões enfrentadas diariamente ${ }^{9}$.

Uma vez que o corpo é o instrumento de expressão na dança ${ }^{10}$, o bailarino vivencia diferentes emoções e transmite a estética da coreografia, numa relação permanente com a sua corporeidade. Na dança clássica, considerada uma manifestação artística com um critério anatômico seletivo, a ênfase é a magreza e linearidade da figura ${ }^{11}$, que requer um alto nível de disciplina, motivação, desempenho excelente e manutenção de uma aparência magra ${ }^{12}$. Já a dança contemporânea, incorpora ${ }^{13}$ muitas diferentes técnicas, movimentos e estilos. Comparado à dança clássica, a dança contemporânea permite ao bailarino uma diversidade de movimentos, menos limitações a determinada técnica, trazendo a necessidade de libertação de formas pré-estabelecidas ${ }^{14}$.

Pode-se dizer que a técnica clássica apresenta normas mais rígidas pelas formas preestabelecidas, e a dança contemporânea preserva mais a individualidade dos bailarinos e, em certos casos, também a sua própria anatomia ${ }^{15}$. A partir do contexto, este estudo se justifica pelo fato dos bailarinos profissionais, dependendo da técnica de dança praticada (clássico ou contemporâneo) se envolverem numa preparação física constante, na qual a demanda por um padrão estético considerando adequado poderá estar relacionada à insatisfação com a imagem corporal.

Sob essa perspectiva, este estudo foi realizado com o objetivo de investigar a satisfação com a imagem corporal e sua relação com a percepção do peso e o índice de massa corporal em bailarinos clássicos e contemporâneos profissionais brasileiros.

\section{MÉTODOS}

Participaram deste estudo descritivo e comparativo, 181 bailarinos profissionais, dos quais 58\% clássicos e 42\% contemporâneos, de ambos os sexos (54,1\% feminino e 45,9\% masculino), com média de idade 24,36 (+/-6,14) anos, de companhias de dança brasileiras. As médias de idade de início na dança, de tempo de dança, tempo diário dedicado à dança e frequência semanal foram respectivamente: 10,59 (+/-5,31) anos, 13,57 (+/-7,33) anos, 6,54 (+/-1,63) horas e 4,39 (+/-,80) vezes.
A seleção dos sujeitos foi de forma não probabilística intencional, uma vez que foram investigados: a) bailarinos atuantes no momento da coleta de dados; b) pertencente a companhias de dança das regiões sul (PR, SC e RS) e sudeste (SP, RJ e MG) do país; c) que aceitaram participar, assinando o termo de consentimento livre e esclarecido e d) que cumpriram o preenchimento completo do questionário e sem rasuras.

A pesquisa foi aprovada pelo Comitê de Ética em Pesquisa da Universidade do Estado de Santa Catarina (Parecer n. 235/2010), que acompanha as normas de Resolução 196/96 do Conselho Nacional de Saúde sobre Pesquisa envolvendo seres humanos.

Na coleta de dados utilizou-se os seguintes Instrumentos de medidas: (a) Questionário anônimo, misto, padronizado, de autopreenchimento, contendo questões sobre as características sociodemograficas e da prática de dança; (b) Escala de nove silhuetas, proposta por Stunkard, segundo recomendações ${ }^{16}$ para aquisição de dados referentes à imagem corporal. Nesta escala são apresentadas nove silhuetas, para cada sexo, com diferentes tamanhos corporais, numeradas da menor (mais magra) para a maior (mais gorda). O (a) avaliado (a) escolhe o número da silhueta que considera mais semelhante a sua aparência corporal real (AC real) e também o número da silhueta que acredita ser mais semelhante à aparência corporal ideal (AC ideal) considerada para sua idade. Para avaliação da satisfação corporal, a nota atribuída subtrai-se da aparência corporal real da aparência corporal ideal, podendo variar de ${ }^{-8}$ até ${ }^{+} 8$. Se essa variação for igual à zero, classifica-se o sujeito como satisfeito; e se diferente de zero, classifica-se como insatisfeito. Caso a diferença seja positiva, é uma insatisfação pelo excesso de peso; e, quando negativa, uma insatisfação pela magreza. A escala é considerada uma medida válida $(r=0,72)$ da imagem corporal em brasileiros ${ }^{17}$; (c) Medidas da massa corporal e estatura foram obtidas por meio de informação autoreferida obtidas através de duas questões autoexplicativas "qual o seu peso e altura atual?", cuja validade tem sido apontada em estudos em diferentes faixas etárias ${ }^{18,19}$. Para classificação do índice de massa corporal (IMC) adotaram-se os critérios da World Health Organization ${ }^{20}$, considerando peso baixo (IMC $\left.<18,5 \mathrm{~kg} / \mathrm{m}^{2}\right)$, normal (IMC 18,50 - 24,99 kg/ m², sobrepeso (IMC $\geq 25 \mathrm{~kg} / \mathrm{m}^{2}$ ) e obeso $\left(\right.$ IMC $\left.\geq 30,00 \mathrm{~kg} / \mathrm{m}^{2}\right)$.

A percepção do peso corporal (PPC) foi avaliada de forma indireta, onde a PPC foi construída pela diferença do peso que os bailarinos referiam como seu peso ideal e o peso atual dele, resultando em uma variável de três categorias: (1) peso ideal menor do que o peso referido - considerado como desejo de pesar menos; (2) peso ideal igual $( \pm 2 \mathrm{~kg})$ ao peso referido - considerado como desejo de permanecer com o mesmo peso; e (3) peso ideal maior do que o peso referido considerado como desejo de pesar mais.

Os questionários foram entregues aos responsáveis pela companhia de dança, os quais foram devidamente instruídos para aplicação do questionário com os bailarinos das suas respectivas companhias de dança. 
Para análise dos dados foi utilizado o software Statistical Package for Social Sciences (SPSS), versão 16.0. Na caracterização dos dados utilizou-se a estatística descritiva (distribuição de frequências (absolutas e relativas), médias, desvio padrão e amplitude de variação). A distribuição dos dados foi averiguada usando-se o teste Kolmogorov-Smirnov (Lilliefors) não sendo constatada aderência a distribuição normal para todas as variáveis investigadas. Optou-se, então, pela estatística não paramétrica (teste do qui-quadrado para verificar a associação entre as variáveis categóricas, a correlação de Spearman para testar as associações e o teste $U$ de Mann-Whitney para comparar as diferenças nas variáveis contínuas. Para todos os procedimentos de análise, adotou-se o nível de significância de 95\% ( $p \leq 0,05)$.

\section{RESULTADOS}

Inicialmente, investigou-se as características antropométricas dos bailarinos profissionais, considerando a técnica de dança (clássico ou contemporâneo). Os resultados estão contidos na tabela 1, a seguir.

Analisando os resultados contidos na tabela 1, constata-se que as medidas antropométricas referidas pelos sujeitos apontaram uma média de massa corporal de 58,54kg (+/-9,72), média de estatura $1,69 \mathrm{~cm}$ $(+/-, 07)$ e média de índice de massa corporal (IMC) $20,37 \mathrm{~kg} / \mathrm{m}^{2}$, considerados adequados de acordo com as referências de normalidade, com uma tendência a um limite inferior. Houve diferenças estatísticas $(p \leq 0,05)$ quanto à massa corporal e o IMC, onde os bailarinos clássicos apresentam valores inferiores que os contemporâneos, em ambas as variáveis, no entanto na variável estatura corporal são equivalentes. Continuando a análise, constatou-se que não houve diferenças estatísticas $(p \leq 0,05)$ para as características antropométricas no gênero masculino, ao contrário do que acontece com as bailarinas.

Na sequência testou-se quais as diferenças significativas entre bailarinos de dança clássica e contemporânea no que se refere o IMC, satisfação com a imagem corporal (SIC) e percepção do peso corporal (PPC) (tabela 2).

Na tabela 2 constatou-se que houve diferenças estatísticas $(p \leq 0,05)$ relativas ao IMC, SIC e PPC entre as técnicas de dança investigadas. Percebe-se que os bailarinos clássicos em sua maioria estão insatisfeitos por excesso de peso (60\%), desejando pesar menos (61\%), embora 80\% destes bailarinos estejam com o IMC próximo do limite inferior, dentro da faixa da normalidade $(18,50$ a 24,99 kg/ m²). Apresentando diferenças significativas em relação aos clássicos a maioria dos bailarinos contemporâneos $(57,9 \%)$ parecem estar satisfeitos com a sua imagem corporal e 48,7\% desejam pesar mais, e 89,5\% tenha um IMC ainda no limite inferior, dentro da faixa da normalidade.

Tabela 1. Características antropométricas de bailarinos profissionais brasileiros.

\begin{tabular}{c|c|c|c|c}
\hline Variáveis & \multicolumn{2}{|c|}{ Técnica de dança } & & \\
\hline & $\begin{array}{c}\text { Clássica } \\
(\mathbf{n}=105)\end{array}$ & $\begin{array}{c}\text { Contemporânea } \\
(\mathbf{n}=\mathbf{7 6})\end{array}$ & $\begin{array}{c}\text { Total } \\
(\mathbf{n}=\mathbf{1 8 1})\end{array}$ & $\mathbf{p}$-valor ${ }^{1}$ \\
\hline & & & & \\
\hline Massa Corporal $(\mathrm{kg})$ & $57,37( \pm 8,92)$ & $60,17( \pm 10,57)$ & $58,54(+/-9,72)$ & $0,014^{*}$ \\
\hline Estatura $(\mathrm{cm})$ & $1,68( \pm, 074)$ & $1,70( \pm, 078)$ & $1,69(+/-, 07)$ & 0,063 \\
\hline IMC $^{2}\left(\mathrm{~kg} / \mathrm{m}^{2}\right)$ & $20,18( \pm 1,91)$ & $20,63( \pm 7,52)$ & $20,37(+/-2,3)$ & $0,029^{*}$ \\
\hline Feminino & & & & \\
\hline Massa Corporal $(\mathrm{kg})$ & $51,78( \pm 4,64)$ & $53,71( \pm 10,11)$ & $52,45(+/-7,04)$ & $0,026^{*}$ \\
\hline Estatura $(\mathrm{cm})$ & $1,64( \pm, 054)$ & $1,64( \pm, 062)$ & $1,64(+/-, 057)$ & 0,840 \\
\hline IMC $\left(\mathrm{kg} / \mathrm{m}^{2}\right)$ & $19,22( \pm 1,41)$ & $19,75( \pm 3,46)$ & $19,40(+/-2,33)$ & $0,005^{*}$ \\
\hline Masculino & & & & \\
\hline Massa Corporal $(\mathrm{kg})$ & $66,09( \pm 6,72)$ & $65,40( \pm 7,70)$ & $65,74(+/-7,20)$ & 0,597 \\
\hline Estatura $(\mathrm{cm})$ & $1,74( \pm, 054)$ & $1,74( \pm, 058)$ & $1,74(+/-, 056)$ & 0,920 \\
\hline IMC $\left(\mathrm{kg} / \mathrm{m}^{2}\right)$ & $21,67( \pm 1,61)$ & $21,35( \pm 1,71)$ & $21,51(+/-1,66)$ & 0,253 \\
\hline
\end{tabular}

${ }^{1}$ Teste de U Man Witney; ${ }^{2}$ IMC: Indice de Massa Corporal. * Diferença estatística significativa $(p \leq 0,05)$.
Aprofundando a análise ao estudo diferenciado dos sexos, verificou-se diferenças estatísticas $(p \leq 0,05)$ entre o IMC das bailarinas contemporâneas $\left(19,75 \mathrm{~kg} / \mathrm{m}^{2}\right)$ com das bailarinas clássicas $\left(19,22 \mathrm{~kg} / \mathrm{m}^{2}\right)$. Em contrapartida não foram encontradas diferenças estatísticas entre os bailarinos do sexo masculino (Figura 1).

Em relação a SIC não houve associações significativas, mas ressalta-se que as bailarinas (clássicas=68,8\%; contemporâneas=44,1\%) apresentam uma insatisfação com a imagem corporal por excesso de peso; já os bailarinos (contemporâneos=66,7\%; clássicos=46,3\%) estão satisfeitos com a sua imagem corporal (Figura 2).

Tabela 2. Associação do índice de massa corporal, satisfação da imagem corporal e percepção do peso corporal em bailarinos profissionais brasileiros.

\begin{tabular}{c|c|c|c|c|}
\hline Variáveis & \multicolumn{4}{|c|}{ Técnica de dança } \\
\hline & $\begin{array}{c}\text { Clássica } \\
\mathbf{n}(\%)\end{array}$ & $\begin{array}{c}\text { Contemporânea } \\
\mathbf{n}(\%)\end{array}$ & $\begin{array}{c}\text { Total } \\
\mathbf{n}(\%)\end{array}$ & p-valor $^{1}$ \\
\hline IMC $^{2}$ & & & & \\
\hline Peso baixo & $21(20,0)$ & $6(7,9)$ & $27(14,9)$ & $0,009^{*}$ \\
\hline Normal & $84(80,0)$ & $68(89,5)$ & $152(84,0)$ & \\
\hline Sobrepeso & - & $2(2,6)$ & $2(1,1)$ & \\
\hline SIC $^{3}$ & & & & \\
\hline Satisfeito & $27(25,7)$ & $44(57,9)$ & $71(39,2)$ & $0,006^{*}$ \\
\hline Insatisfeito & & & & \\
\hline EP $^{4}$ & $63(60,0)$ & $19(25,0)$ & $82(45,3)$ & \\
\hline EM $^{5}$ & $15(14,3)$ & $13(17,1)$ & $28(15,5)$ & \\
\hline PPC $^{6}$ & & & & \\
\hline Desejo de pesar menos & $64(61,0)$ & $34(44,7)$ & $98(54,1)$ & $0,004^{*}$ \\
\hline Desejo de permanecer com & $15(14,2)$ & $5(6,6)$ & $20(11,1)$ & \\
\hline o mesmo peso & $26(24,8)$ & $37(48,7)$ & $63(34,8)$ & \\
\hline Desejo de pesar mais & $105(100,0)$ & $76(100,0)$ & $181(100,0)$ & \\
\hline Total & & & \\
\hline
\end{tabular}

Teste do Qui-quadrado; ${ }^{2}$ IMC: Indice de Massa Corporal; ${ }^{3}$ Satisfação com a Imagem Corporal; ; EP:Excesso de Peso; ${ }^{5}$ EM:Excesso de Magreza; ${ }^{6}$ PPC: Percepçăo do Peso Corporal; * Diferença estatística significativa $(p \leq 0,05)$.

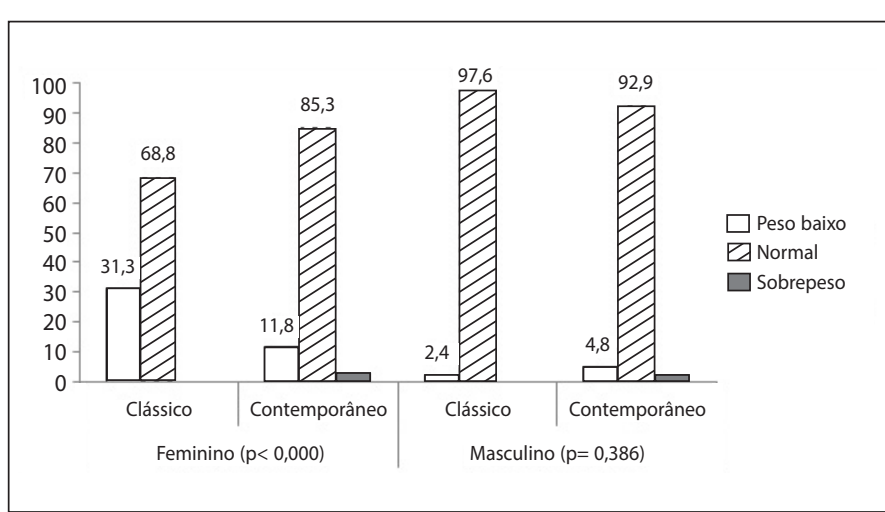

Figura 1. IMC dos bailarinos clássicos e contemporâneos, segundo o sexo.

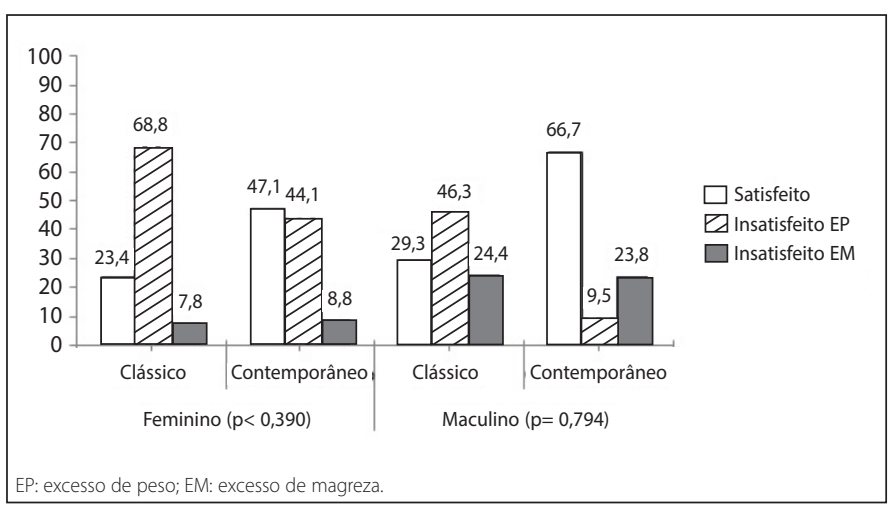

Figura 2. Satisfação com imagem corporal dos bailarinos clássicos e contemporâneos, segundo o sexo. 
Na figura 3 averiguou-se diferenças estatísticas $(p \leq 0,05)$ na PPC em ambos os sexos, demonstrando que as bailarinas desejam pesar menos (clássicas=75\%; contemporâneas $=64,7 \%$ ) e os bailarinos (contemporâneos=69\%; clássicos=43,9\%) desejam pesar mais.

Por fim, testou-se a existência ou não de correlações entre a SIC e o IMC e SIC e PPC nas técnicas de dança clássica e contemporânea. Os resultados constam na tabela 3, a seguir.

Analisando-se os resultados dispostos na tabela 3, verifica-se que de um modo geral, os valores das correlações (rs) entre a SIC e o IMC e entre a SIC e o PPC foram baixos em ambas as técnicas de dança (clássica e contemporânea). Somente houve correlação significativa entre SIC com IMC na dança contemporânea, porém inversa, ou seja quanto menor é a percepção de peso maior a satisfação com a imagem corporal. No entanto, no grupo de bailarinos clássicos esta correlação não se verifique, mostrando que, apesar de muito magros os bailarinos continuam insatisfeitos com a sua aparência.

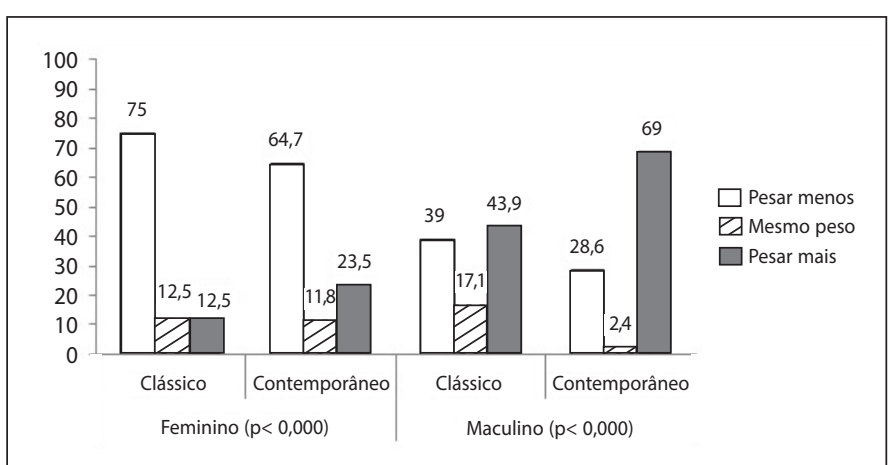

Figura 3. Percepção do peso corporal dos bailarinos clássicos e contemporâneos, segundo o sexo.

Tabela 3. Comparação da satisfação com a imagem corporal com o índice de massa corporal e percepção do peso corporal em bailarinos profissionais brasileiros.

\begin{tabular}{c|c|c|c}
\hline Técnica de Dança & Variáveis & rs & p-valor $^{1}$ \\
\hline \multirow{2}{*}{ Clássica } & SIC $^{2} \times \mathrm{IMC}^{3}$ & 0,330 & 0,741 \\
\cline { 2 - 4 } & $\mathrm{SIC} \times \mathrm{PPC}^{4}$ & $-0,152$ & 0,0121 \\
\hline \multirow{2}{*}{ Contemporânea } & $\mathrm{SIC} \times \mathrm{IMC}$ & $-0,281$ & $0,014^{*}$ \\
\cline { 2 - 4 } & $\mathrm{SIC} \times \mathrm{PPC}$ & 0,006 & 0,958 \\
\hline
\end{tabular}

'Correlação de Speerman; ${ }^{2}$ SIC: Satisfação com Imagem Corporal; ${ }^{3}$ MC: Indice de Massa Corporal; ${ }^{4}$ PPC: Percepção do Peso Corporal. * Correlaçäo estatisticamente significativa $(p \leq 0,05)$.

\section{DISCUSSÃO}

Com relação às características antropométricas dos bailarinos (tabela 1), os resultados deste estudo revelam que os índices médios de IMC $\left(20,37 \mathrm{~kg} / \mathrm{m}^{2}\right)$ estão dentro da referência da normalidade para a população $\left(18,5\right.$ a $\left.24,99 \mathrm{~kg} / \mathrm{m}^{2}\right)$ segundo a $W_{H} \mathrm{HO}^{20}$. Estas médias de IMC, são semelhantes ao valor da média esperada $\left(19,9 \mathrm{~kg} / \mathrm{m}^{2}\right)$ para bailarinos proposta por Heyward e Stolarczyk ${ }^{21}$. Resultados semeIhantes a estes também foram encontrados em estudos anteriores envolvendo bailarinos clássicos brasileiros ${ }^{22}$ onde o índice médio de IMC foi de 20,30 kg/m². Da mesma forma, índices médios de IMC foram encontrados em estudos com bailarinos clássicos europeus $\left(20,55 \mathrm{~kg} / \mathrm{m}^{2}\right)$, gregos $\left(19,9 \mathrm{~kg} / \mathrm{m}^{2}\right)$ e americanos $\left(20,1 \mathrm{~kg} / \mathrm{m}^{2}\right)^{23-25}$. Isto significa que apesar do nosso estudo incluir bailarinos contemporâneos, os índices médios que encontramos não se diferenciam muito dos estudos anteriores relativos apenas a bailarinos clássicos, embora o nosso estudo tenha verificado diferença significativa entre ambas as técnicas.

O fato é que a forma física é uma das maiores demandas artísticas da dança, onde o peso das bailarinas clássicas é em média 12\% a 15\% a menos dos valores referenciais da normalidade, porém nas companhias de dança moderna permite-se uma maior variedade de formas ${ }^{4}$, como nosso estudo parece indicar.

No estudo verificou-se que as bailarinas clássicas são mais leves e magras do que as contemporâneas, indicando necessidade de corpos longilíneos na dança clássica do que na contemporânea. Da mesma forma, ${ }^{26}$ descobriram que entre os dois grupos (clássicos e modernos) que os dançarinos da técnica de dança moderna, eram mais velhos, mais pesados e tinham uma composição corporal mais elevada do que a composição corporal de bailarinos clássicos. Já no sexo masculino não foram encontradas diferenças para as características antropométricas, e acredita-se que o corpo do bailarino mais musculoso no clássico permite a aceitação de silhuetas menos magras que a das bailarinas, e assim, o bailarino do sexo masculino tem maior possibilidade de escolher a técnica praticada, independentemente das suas características antropométricas.

No que se refere à imagem corporal e percepção do peso, os bailarinos clássicos do estudo estão insatisfeitos por excesso de peso, desejando pesar menos. O impacto desta situação revela-se no desejo por um corpo mais magro, e a discrepância entre peso real e o ideal leva a um estado de constante insatisfação com o próprio corpo. Isto possivelmente poderá causar distorções dessa imagem²7,28. Nesta premissa, Ribeiro e Veiga ${ }^{29}$ concluíram em seu estudo, que mesmo possuindo corpos magros, muitos bailarinos estão insatisfeitos, com seu corpo e desejam ser mais magros devido ao rígido padrão estético exigido nessa profissão. No estudo de Haas, Garcia e Bertoletti ${ }^{30}$ dentre os três grupos estudados (balé clássico e jazz), observou- se níveis de insatisfação e distorção da imagem corporal, não havendo diferenças significativas entre eles. Os resultados indicavam que, independente da modalidade, qualquer manifestação artística na forma de dança traria consigo a preocupação do bailarino com sua estética corpora| ${ }^{8,9}$. Ao contrário, o nosso estudo revelou que os bailarinos contemporâneos possuem em sua maioria níveis de satisfação muito superiores aos dos bailarinos clássicos.

Os bailarinos contemporâneos desejam pesar mais, sobretudo nos bailarinos de gênero masculino. Isto poderá estar relacionado ao desejo de desenvolver a massa muscular que aparece muito valorizada na sociedade atual. Contrariamente aos resultados do estudo, Clabaugh e Morling ${ }^{31}$ apontaram que os dançarinos modernos são insatisfeitos com seus corpos $(39,6 \%)$ como os de balé $(45 \%)$. Isto pode indicar que o universo da dança contemporânea está atualmente mais aberto à diversidade corporal que a dança moderna. Sabe-se que a dança contemporânea incorpora diversas técnicas, movimentos e estilos e, comparado ao balé, a dança contemporânea é caracterizada por uma maior variedade de exigências técnicas impostas por coreógrafos durante a performance ${ }^{32}$. Sendo assim, é natural que os bailarinos contemporâneos por executarem um repertório mais diversificado e serem melhor aceitos na sua diversidade corporal, estejam satisfeitos com sua imagem corporal.

Não foram encontradas correlação entre a SIC e PPC/IMC na maioria dos bailarinos investigados, mas pode-se verificar que a magreza dos bailarinos clássicos não parece influenciar a sua satisfação com imagem corporal, exceto no caso dos bailarinos contemporâneos onde se constatou uma relação entre o IMC mais baixo e uma maior SIC. Os resultados desta investigação não vão de encontro dos achados de Pollatou et al. ${ }^{32}$ que indicam uma associação entre satisfação corporal e preocupação com excesso de peso. Acredita-se que a diferença encontrada no nosso estudo poderá ser resultados de atitudes estéticas menos estereotipadas e mais voltadas para a diversidade corporal. Neste sentido, podendo estar relacionada a atitudes menos arbitrárias dos diretores e coreógrafos das companhias relativamente 
ao peso dos bailarinos, que demonstraram em nosso estudo aceitar melhor a sua individualidade.

O resultado encontrado nesta pesquisa demonstra que independentemente da técnica de dança, os bailarinos apresentam percepções diferentes da sua imagem. Verificou-se que nos bailarinos do sexo de dança clássica e contemporânea não se encontram diferenças significativas a nível de peso, estatura ou IMC, enquanto uma maior percentagem de bailarinas clássicas têm IMC abaixo da média do que as contemporâneas. No entanto estas bailarinas, com IMC mais alto têm também uma maior satisfação com a sua imagem, o que se compreende se atentarmos a que a dança clássica é muito mais exigente em relação à magreza das bailarinas do que acontece na dança contemporânea, em que se aceita com mais facilidade um corpo fora dos padrões estéticos clássicos.

Os bailarinos clássicos do estudo estão na sua maioria insatisfeitos por excesso de peso, desejando pesar menos, enquanto os bailarinos de contemporâneo estão em grande número satisfeitos com a sua imagem corporal e um grupo significativo desejaria pesar mais. Isto acontece sobretudo nos bailarinos do sexo masculino, e estará certamente relacionado ao desejo de desenvolver a massa muscular que aparece muito valorizada na sociedade atual. Somos levadas a pensar que os padrões estéticos dos bailarinos contemporâneos estão eventualmente mais perto dos padrões da sociedade atual que o dos bailarinos clássicos.

\section{CONCLUSÃO}

Considera-se relevante a intensificação de estudos sistemáticos sobre a dança e imagem corporal, tanto no Brasil quanto em outros países, a fim de prevenir sérias consequências à saúde dos bailarinos.

Finalmente, é possível destacar que, no presente estudo, os bailarinos profissionais clássicos e contemporâneos brasileiros possuem um IMC considerado adequado, de acordo com as referências de normalidade, com uma tendência a um limite inferior. A técnica de dança adotada pelos bailarinos pode levar a opiniões e visões estereotipadas do corpo, e as diferenças encontradas refletem uma maior abertura à diversidade corporal no meio da dança contempoâneo que na dança clássica.

Todos os autores declararam não haver qualquer potencial conflito de interesses referente a este artigo.

\section{REFERÊNCIAS}

1. Tavares MCC. Imagem corporal e dança. Rev Conexões. 2001;1 (6):10-22.

2. Saikali CJ, Soubhia CS, Scalfo BM, Cordás, A. Imagem corporal nos transtornos alimentares. Rev Psiquiatr Clin. 2004; 31:164-6.

3. Damasceno VO, Lima JRP, Vianna JM, Vianna VRA, Novaes JS. Tipo físico ideal e satisfação com a imagem corporal de praticantes de caminhada. Rev Bras Med Esporte. 2005; 11(3):181-86.

4. Bolling CS. Pinheiro TMM. Bailarinos profissionais e saúde: uma revisão de literatura. Rev Med Minas Gerais. 2010;20:(2 Supl 2): S75-S83.

5. Tiggemann M, Slater A. A test of objectification theory in former dancers and non dancers. Psychol Women Q. 2001;25:57-64.

6. Simas JN, Guimarães AC. Ballet Clássico e Transtornos Alimentares. Rev Edu Fís UEM. 2002;13(2):119-26.

7. Dotti A, Fioravanti M, Balotta M, Tozzi F, Cannella C, Lazzari R. Eating behavior of ballet dancers. Eat Weight Disord. 2002;7(1):60-7.

8. Ackard DM, Henderson JB, Wonderlich AL. The association between childhood dance participation and adult disordered eating related psychopathology. J Psychosom Res. 2004;57(5):485-90.

9. Ravaldi C, Vannacci A, Bolognesi E, Mancini S, Faravelli C, Ricca V. Gender role, eating disorder symptoms, and body image concern in ballet dancers. J Psychosom Res. 2006;61(4):529-35.

10. Angioi M, Metsios GS, Twitchett E, Koutedakis Y.Wyon M. Association between selected physical fitness parameters and esthetic competence in contemporary dancers. J Dance Med Sci 2009;13(4):115-23.

11. Hamilton WG, Hamilton LH, Marshall P, Molnar M. A profile of the musculoskeletal characteristics of elite professional ballet dancers. Am J Sports Med. 1992;20(3):267-73.

12. Eliakim A, Ish-Shalom S, Giladi A, Falk B, Constantini N. Assessment of body composition in ballet dancers: correlation among anthropometric measurements, bio-electrical impedance analysis, and dual-energy X-ray absorptiometry. Int J Sports Med. 2000;21 (8):598-601.

13. Weiss DS, Shah S, Burchette RJ. A profile of the demographics and training characteristics of professional modern dancers. J Dance Med Sci. 2008;12(2):41-6.

14. Assumpção ACR. O balé clássico e a dança contemporânea na formação humana: caminhos para a emancipação. Rev Pensar a Prática. 2003;6:1-19.

15. Silva AH, Bonorino KC. IMC e Flexibilidade de Bailarinas de Dança Contemporânea e Clássica. Fit Perform J 2008; 7:48-51.

16. Gardner RM, Friedman BN, Jackson NA. Methodological concerns when using silhouettes to measure body image. Percept Mot Skills. 1998;86(2):387-95
17. Scagliusu FB, Alvarenga M, Polacow VO, Cordás TA, Queiroz GKO, Coelho D, et al. Concurrent and discriminant validity of the Stunkard's figure rating scale adapted into Portuguese. Appetite. 2006;47(1):77-82.

18. Silveira EA, Araújo CL, Gigante DP, Barros AJD, Lima MS. Validação do peso e altura referidos para o diagnóstico do estado nutricional em uma população de adultos no Sul do Brasil. Cad Saúde Pública. 2005:21 (1):235-45.

19. Rech CR, Petroski EL, Babel Júnior RJ, Soares MR. Concordância entre as medidas de peso e estatura mensuradas e auto-referidas para o diagnóstico do estado nutricional de idosos residentes no sul do Brasil. Rev Bras Med Esporte. 2008;14(2):126-31.

20. World Health Organization (WHO). Global Database on Body Mass Index. Disponível em: http://apps. who.int/bmi/index.jsp?introPage=intro_3.html. Acessado em 1/3/2012.

21. Heyward VH, Stolarczyk LM. Avaliação da composição corporal aplicada. São Paulo: Manole; 2000

22. Frasson VB, Rassier DE, Herzog W, Vaz MA. Dorsiflexor and plantarflexor torque-angle and torquevelocity relationships of classical ballet dancers and volleyball players. Braz J Biomech. 2007;8(14):31-7.

23. Wyon MA, Allen N, Angioi M, Nevill AM, Twitchett E. Anthropometric factors affecting vertical jump heigh in ballet dancers. Int A Dance Med Sci 2006, 10: 106-10.

24. Yannakoulia M, Keramopoulos A, Tsakalakos N, Matalas AL. Body composition in dancers: the bioelectrical impedance method. Med Sci Sports Exerc. 2000;32: 228-34.

25. Tsunawake N, Tahara Y, Moji K, Muraki S, Minowa K, Yukawa K. Body composition and physical fitness of female volleyball and basketball players of the Japan inter-high school championship teams. J Physiol Anthropol Appl Human Sci. 2003;22:195-201

26. Potter AB, Lavery ES, Bell RA. Body fat and body mass index measurements in preprofessional dance students. A comparison of formulas. Med Probl Perform Art. 1996;11:43-6.

27. Smolak L, Murnen S, Ruble AE. Female athletes and eating problems. A meta-analysis. Int J Eat Disord. 2000;27(4):371-80.

28. Thompson AM, Chad KE. The relationship of social physique anxiety to risk for developing and eating disorder in Young females. J Adolesc Health. 2002;31(2):183-9.

29. Ribeiro LG, Veiga GV. Imagem corporal e comportamentos de risco para transtornos alimentares em bailarinos profissionais. Rev Bras Med Esporte. 2010; 16:99-102.

30. Haas NA, Garcia ACD, Bertoletti J. Imagem corporal e bailarinas profissionais. Rev Bras Med Esporte 2010; 16: 182-5.

31. Clabaugh A, Morling B. Stereotype accuracy of ballet and modern dancers. J Soc Psychol. 2004;144(1):31-48

32. Pollatou E, Bakali N, Theodorakis Y, Goudas M. Body image in female professional and amateur dancers. Res Dance Educ. 2010;11:131-7. 